\title{
Characterization of Lipooligosaccharides from Haemophilus ducreyi Containing Polylactosamine Repeats
}

\author{
Birgit Schilling and Bradford W. Gibson* \\ Buck Institute for Age Research, Novato, California, USA \\ Melanie Filiatrault and Anthony A. Campagnari \\ Infectious Disease Institute, State University of New York, Buffalo, New York, USA
}

\begin{abstract}
Haemophilus ducreyi, a gram-negative human mucosal pathogen, is one of the principal causes of genital ulcer disease. The lipooligosaccharides (LOS) of these bacteria are considered to be a major virulence factor and have been implicated in the adherence and invasion of $H$. ducreyi to several human cell types. An isogenic heptosyltransferase-III knockout strain (waaQ) was recently constructed from $\mathrm{H}$. ducreyi 35000 wild-type strain and immunochemical and molecular weight data of the isolated LOS suggested the presence of poly-N-acetyllactosamine (LacNAc) (Filiatrault et al., Infect. Immun. 2000, 68, 3352-3361). In this present study, the structures of these novel LOS-glycoforms were characterized by matrix-assisted laser desorption/ionization (MALDI) and electrospray ionization (ESI) mass spectrometry in combination with exoglycosidase digestion. Detailed structural information was obtained for the oligosaccharide (OS) portions of these LOS showing between one to five linear LacNAc repeats on the non-reducing terminus of the main oligosaccharide branch. When grown on solid media, the organism produced LacNAc repeats that were further modified by the addition of sialic acid. Enzymatic digestion with $\beta$-galactosidase, $\beta$-N-acetylhexosaminidase, and neuraminidase type VI-A yielded truncated glycoforms consistent with a polyLacNAc structure capped at various end points with sialic acid. ESI-MS/MS mass spectrometry on a quadrupole time-offlight instrument was particularly effective in obtaining detailed structural information on the least abundant, high-mass glycoforms. Although LOS containing terminal di-LacNAc have been reported, this is the first time to our knowledge that a linear polyLacNAc structure has been characterized in bacteria. (J Am Soc Mass Spectrom 2002, 13, 724-734) (c) 2002 American Society for Mass Spectrometry
\end{abstract}

$\mathrm{H}$ aemophilus ducreyi is a gram-negative bacterium which causes genital ulcer disease or chancroid. Although chancroid is uncommon in the United States, it is prevalent in many developing countries worldwide [1]. Chancroid has also been shown to be a significant risk factor for transmission of human immunodeficiency virus (HIV) [2-4]. Recently, the number of $H$. ducreyi clinical isolates with antibiotic resistance has increased [1, 5], suggesting that this sexually transmitted disease is far from contained and represents a growing threat to world health.

Although several outer-membrane or secreted components of $H$. ducreyi have been suggested as contributing to the pathogenesis and virulence of this organism, our laboratory has investigated the structures and

Published online April 26, 2002

Address reprint requests to Dr. B. W. Gibson, Buck Institute for Age Research, 8001 Redwood Blvd., Novato, CA 94945, USA. E-mail: bgibson@buckinstitute.org

*Also at the Department of Pharmaceutical Chemistry, University of California, San Francisco, San Francisco, CA 94143-0446. possible functions of outer-membrane lipooligosaccharide (LOS). LOS is considered to be a major virulence factor and has been implicated as a cytotoxin [6] as well as contributing in the adherence and invasion of $H$. ducreyi to cultured human foreskin fibroblasts and keratinocytes [7, 8]. Structures of major LOS-glycoforms from several wild type strains have been identified and shown to contain terminal epitopes that mimic human glycoconjugates [9-12]. As shown in Figure 1, bacterial lipooligosaccharides consist of a Lipid A and oligosaccharide moiety, the latter containing a conserved oligosaccharide core region that is substituted with one or more oligosaccharide branches. The major LOS-glycoform from the prototype $H$. ducreyi 35000 strain has been shown by NMR and mass spectrometry to contain a terminal N-acetyllactosamine (LacNAc) disaccharide, Gal $\beta 1 \rightarrow 4$ GlcNAc $\beta 1$, that is partially modified by the addition of sialic acid (N-acetylneuraminic acid, or NeuAc) to form NeuAc $\alpha 2 \rightarrow 3 \mathrm{Gal} \beta 1 \rightarrow 4 \mathrm{GlcNAc}$ [11, 13]. In addition, Melaugh et al. [10] reported that a di-LacNAc glycoform was also present at very low 


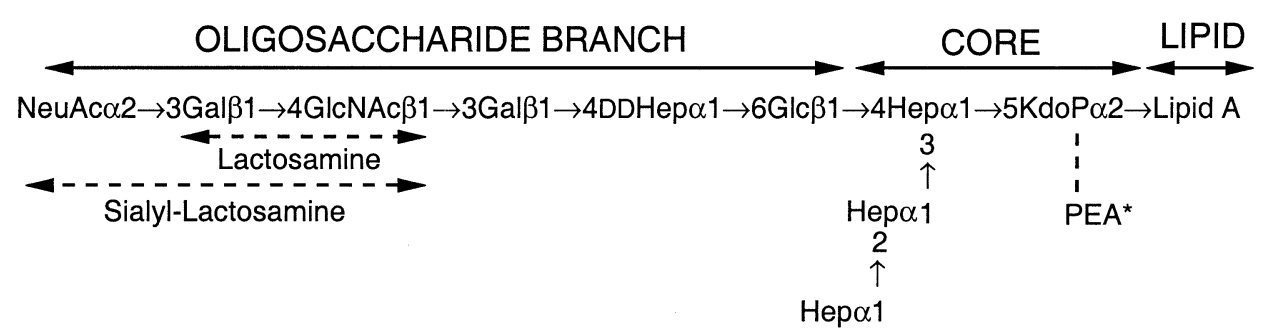

Figure 1. Bacterial lipooligosaccharides contain a Lipid A and an oligosaccharide moiety which includes a conserved oligosaccharide core and one or more oligosaccharide branches added to the core structure. The major LOS-glycoform from the prototype $H$. ducreyi 35000 strain contains a terminal $\mathrm{N}$-acetyllactosamine (LacNAc) disaccharide, $\mathrm{Gal} \beta 1 \rightarrow 4 \mathrm{GlcNAc} \beta 1$, that is partially modified by the addition of sialic acid.

abundances in strain 35000. A similar observation was reported by Schweda et al. [14] for several clinical isolates. The di-LacNAc pathway appears to compete with a more dominant biosynthetic pathway that adds sialic acid, effectively capping the extension of the oligosaccharide branch [10]. Although a di-LacNAc structure has also been suggested to be present in the related mucosal pathogen Neisseria gonorrhoeae [15], little structural evidence has been presented other than molecular mass to support these assignments, in part due to the very low abundance of these extended LOS-glycoforms under in vitro growth conditions. Moreover, to the best of our knowledge, there have been no reports of LacNAc structures extended beyond two repeats to form polylactosamine (polyLacNAc) LOS-glycoforms in bacteria despite numerous reports of analogous structures in eukaryotes [16-18].

Over the past five years, several LOS-specific glycosyltransferases have been identified and gene knockout mutants constructed from the prototype $H$. ducreyi wild-type strain 35000 . To verify the specificity of these glycosyltransferases, structural studies of the resulting altered LOS-glycoforms have been carried out [13, 19]. In a recent study, an isogenic heptosyltransferase-III knockout strain (35000hep-) was constructed by Filiatrault et al. [20] in which immunochemical and molecular mass data suggested the presence of several unusual structural features. Specifically, a series of high molecular weight glycoforms $\left(\mathrm{M}_{r}>3000\right)$ were observed by SDS-PAGE which reacted with a monoclonal antibody (3F11) that recognizes LOS containing terminal LacNAc or paragloboside [21]. Moreover, the masses of the glycoforms as determined by MALDI-MS indicated that these glycoforms were formed by mutiple additions of alternating Hex and HexNAc residues, consistent with a polyLacNAc structure. To provide structural support for these tentative assignments, we have carried out an extensive characterization of these novel LOS-glycoforms using a combination of mass spectrometric methods (MS and MS/MS) and exoglycosidase digestion with $\beta$-galactosidase, $\beta$-N-acetylhexosaminidase, and neuraminidase type VI-A. These data now show that these high molecular weight LOS-glycoforms contain up to five repeating LacNAc units on a single oligosaccharide branch that can also undergo substitution with sialic acid under solid phase growth conditions.

\section{Experimental}

\section{Materials}

$\mathrm{N}$-Acetylneuraminic acid was obtained from Sigma (St. Louis, MO). GC Medium Base, brain heart infusion, and hemoglobin were obtained from DIFCO (Detroit, MI); inactivated fetal bovine serum (FBS) was obtained from the Cell Culture Facility at UCSF; IsoVitaleX was purchased from Becton Dickinson (Franklin Lakes, N.J.). All reagents used in chemical reactions were obtained from commercial suppliers and used without further purification unless otherwise noted.

\section{Methods}

Bacterial strains and culture conditions. H. ducreyi strains were cultured at $35^{\circ} \mathrm{C}$ in $5 \% \mathrm{CO}_{2}$ on chocolate agar plates or in brain heart infusion broth as previously described [22]. When needed, chocolate agar plates were supplemented with Kanamycin $(20 \mu \mathrm{g} / \mathrm{ml})$, chloramphenicol $(1 \mu \mathrm{g} / \mathrm{ml})$, and/or 5-bromo-4-chloro-3-indolyl- $\beta$-D-galactopyranoside (X-Gal; $40 \mu \mathrm{g} / \mathrm{ml}$ ). The construction of these isogenic mutants was previously described [20].

Preparation of LOS. LOS from proteinase K-treated whole-cell lysates was resolved by sodium dodecyl sulfate polyacrylamide gel electrophoresis (SDS-PAGE) on a $14 \%$ acrylamide gel and visualized by silver staining [22, 23]. Western blot analysis was performed by transferring LOS to a PVDF membrane (Millipore, Bedford, MA) using a previously described procedure [24]. LOS for structural analysis was extracted by using the modified hot phenol-water procedure from bacteria that were grown overnight in $1.6 \mathrm{~L}$ of broth and dried [25-27]. In each case, approximately $0.5-1.0 \mathrm{mg}$ of LOS was converted to the O-deacylated LOS-glycoforms by treatment with hydrazine $\left(37^{\circ} \mathrm{C}, 30 \mathrm{~min}\right)$ [28], a process that increases solubility and makes the LOS more amenable to mass spectrometric analysis [9]. In addition, a portion of the O-deacylated LOS was further 
treated with $48 \%$ aqueous hydrofluoric acid (HF) (at $4{ }^{\circ} \mathrm{C}$ for $12 \mathrm{~h}$ ) to remove phosphoethanolamine (PEA) and phosphate $(\mathrm{P})$ groups [10]. Finally, an oligosaccharide fraction of strain 35000hep- (broth) was prepared by hydrolysis of the crude LOS $(0.5-1.0 \mathrm{mg})$ in a solution of $1 \%$ acetic acid $(350 \mu \mathrm{l})$ at $100{ }^{\circ} \mathrm{C}$ for $2 \mathrm{~h} \mathrm{[10].}$ The oligosaccharide fractions were then separated from the Lipid A fraction by centrifugation $\left(5000 \times g, 4{ }^{\circ} \mathrm{C}\right)$ and purified by size exclusion chromatography using two $300 \times 7.8 \mathrm{~mm}$ BioSelect SEC 125-5 columns (BioRad) connected in series. Samples were eluted in $50 \mathrm{mM}$ pyridinium acetate ( $\mathrm{pH}$ 5.2) at a flow rate of $1 \mathrm{~mL} \mathrm{~min}^{-1}$ and collected in $0.5-1 \mathrm{~mL}$ fractions. Alternatively, an oligosaccharide fraction was prepared from strain 35000hep - (plate) under milder conditions that retains terminal sialic acid residues. This method consisted of hydrolyzing the $O$-deacylated LOS in a $100 \mathrm{mM}$ ammonium acetate solution $(\mathrm{pH} 4.5)$ at $80^{\circ} \mathrm{C}$ for $1 \mathrm{~h}$. The crude reaction mixture was then directly analyzed by mass spectrometry.

Exoglycosidase digestion. To determine the identity and linkages of terminal sugars in these LOS preparations, $O$-deacylated LOS was subjected to treatment with specific exoglycosidases and re-analyzed by MALDIMS. Exo-glycosidases used were $\beta$-galactosidase from Jack bean meal ( $\beta$-Galase, highly specific for cleavage of $\beta$-anomeric glycosidic bonds; hydrolysis of $\beta 1-6$ and $\beta 1-4$ linked Gal-GlcNAc clearly favored over the corresponding $\beta 1-3$ bonds with relative hydrolysis rates, 100:75:1, respectively), $\beta$ - $\mathrm{N}$-acetylhexosaminidase from Jack bean meal ( $\beta$-HexNase, highly specific for cleavage of $\beta$-anomeric glycosidic bonds such as $\beta 1-2,3,4$ and 6 linked GlcNAc and GalNAc residues), and/or neuraminidase type VI-A from Clostridium perfringens attached to beaded agarose (NANase, specific for cleavage of terminal NeuAc). $\beta$-galactosidase and $\beta$-Nacetylhexosaminidase were purchased from Oxford GlycoScience (Oxford, UK), neuraminidase was purchased from Sigma (St. Louis, MO). Approximately 10 $\mu \mathrm{g}$ of $\mathrm{O}$-deacylated LOS was dissolved in $25 \mu \mathrm{l}$ of 50 $\mathrm{mM}$ ammonium acetate buffer ( $\mathrm{pH}$ 4.5). Digestions with alternating enzymes were performed sequentially [15]. For each digestion step, $\mathrm{O}$-LOS were incubated at $37^{\circ} \mathrm{C}$ for $24 \mathrm{~h}$ using $\beta$-galactosidase $(0.8$ units $/ \mathrm{ml}$ ) or $\beta-\mathrm{N}$ acetylhexosaminidase $(50$ units $/ \mathrm{ml})$, respectively, whereas a shorter incubation time was chosen for neuraminidase treatment $\left(37^{\circ} \mathrm{C}\right.$ for $3 \mathrm{~h}, 2$ units $\left./ \mathrm{ml}\right)$. Each digestion step was terminated after completion by heating the reaction mixture for $2 \mathrm{~min}$ at $100{ }^{\circ} \mathrm{C}$ to deactivate the enzyme. An aliquot was taken for MS analysis and subsequently an alternating enzyme was added and the reaction was incubated.

Matrix-assisted laser desorption/ionization mass spectrometry. The O-deacylated LOS, HF-treated O-deacylated LOS, and acid released oligosaccharide fractions from the mutant and parental 35000 strain were all analyzed by MALDI-MS. An Applied Biosystems (Fra- mingham, MA) Voyager DE or a Voyager DESTR time-of-flight mass spectrometer were used to analyze the various LOS preparations as previously described [9]. Mass spectra were run in both the positive- and negative-ion mode and under post-source decay conditions (PSD) [29]. Both instruments were equipped with a nitrogen laser $(337 \mathrm{~nm})$ and operated under delayed extraction conditions [30]; delay time was 100-175 ns, grid voltage was $92-94 \%$ (linear mode) and $70-75 \%$ (reflectron mode), respectively, of full acceleration voltage $(20-30 \mathrm{kV})$. In PSD mode, precursor ions were selected by timed-ion selection and the resulting fragmentation products were focused by adjusting the reflectron voltage in 9-11 steps. The acquired segment spectra were then stitched together to obtain the composite PSD spectrum; PSD spectra were smoothed using a Gaussian algorithm causing fragment ions to appear as average masses. Samples were purified and desalted by drop dialysis using a $0.025 \mu \mathrm{m}$ nitrocellulose membrane and/or by anion exchange Zip Tips ${ }_{\mathrm{AX}}$ (Millipore). Approximately $0.1-0.2 \mu \mathrm{g}$ of O-deacylated LOS was mixed with $1 \mu \mathrm{l}$ of a $320 \mathrm{mM}$ 2,5-dihydroxybenzoic acid (DHB) in 4:1 acetone/water (vol/vol) containing 175 $\mathrm{mM}$ 1-hydroxyisoquinoline (HIC) [31], desalted with cation-exchange resin beads (DOWEX, 50X, $\mathrm{NH}_{4}^{+}$) and then air dried on a stainless steel target. Spectra were acquired, averaged and mass calibrated with an external calibrant consisting of an equimolar mixture of angiotensin II, bradykinin, LHRH, bombesin, $\alpha$-MSH (CZE mixture, Bio-Rad) and ACTH 1-24 (Sigma, St. Louis, MO). Typically, 20-50 laser shots were used to record each linear spectrum, or spectral segment if PSD conditions were used.

Electrospray Ionization Mass Spectrometry. Oligosaccharides and HF-treated $O$-deacylated LOS were further analyzed in positive-ion mode on a quadrupole orthogonal time-of-flight mass spectrometer (QSTAR, PE Sciex, Concorde, Canada). Samples were dissolved in a 1:1 solution of $100 \mathrm{mM} \mathrm{NH}_{4} \mathrm{OAc}(\mathrm{pH} 4.5)$ and acetonitrile and loaded into a Protana nanospray tip. The nanospray needle voltage was typically $1200-1300 \mathrm{~V}$. Mass spectra (ESI-MS) and tandem mass spectra (ESIMS/MS) of O-deacylated LOS and of HF-treated Odeacylated LOS were recorded in positive-ion mode. In MS mode a resolution of 12,000-15,000 FWHM was achieved, which allowed unambiguous determination of the charge state. For collision induced dissociation (CID) MSMS, the mass window for precursor ion selection of the quadrupole mass analyzer was generally set to $\pm 1 \mathrm{~m} / \mathrm{z}$. The selected ions were fragmented in a collision cell using air as collision gas and analyzed in the orthogonal TOF operating at an acceleration potential of $4 \mathrm{kV}$. Spectra were calibrated using MS/MS fragment-ions of a glufibrino peptide standard (EGVNDNEEGFFSAR, $\mathrm{m} / \mathrm{z}$ at 187.0719 [B $\mathrm{B}_{2}$-ion], and $\mathrm{m} / \mathrm{z}$ at $1285.4995\left[\mathrm{Y}_{11}\right.$-ion]) providing a mass accuracy of $\leq 50 \mathrm{ppm}$. 


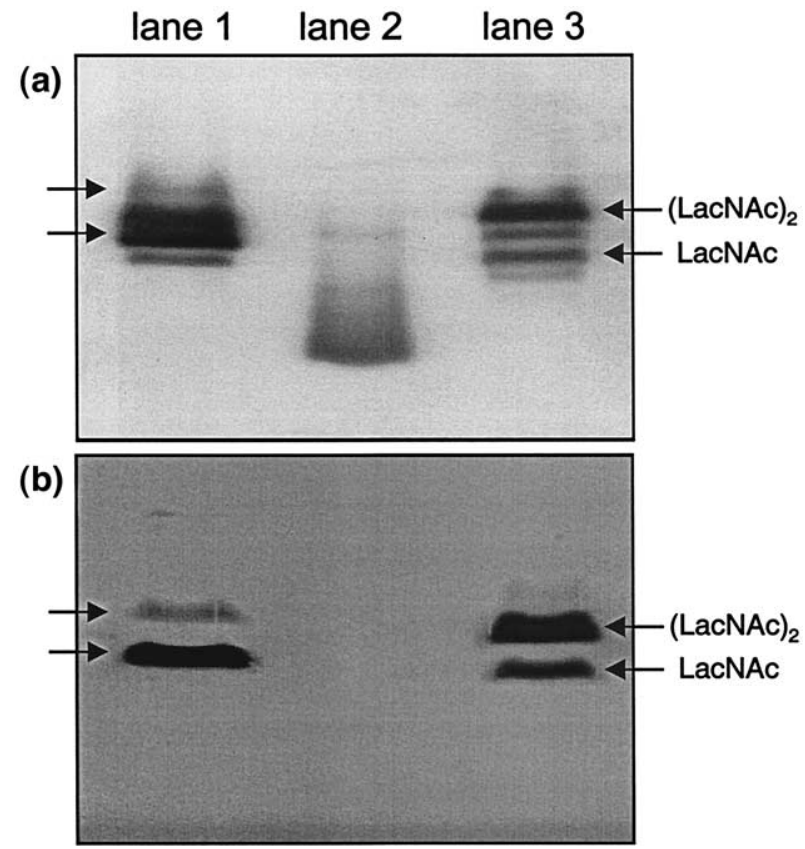

Figure 2. A composite of a silver stained SDS-PAGE gel (panel a) and the corresponding Western Blot (panel $\mathbf{b}$ ). The gel contains LOS from $H$. ducreyi wild-type strain 35000 (lane 1), double knockout mutant strain HD35000R (lane 2), and heptosyltransferase knockout mutant 35000hep- (lane 3). For Western staining the monoclonal antibody 3F11 was used. This antibody which was originally generated against LOS from $N$. gonorrhoeae has specificity for terminal LacNAc.

\section{Results and Discussion}

The lipooligosaccharides from the isogenic waa $\mathrm{mu}-$ tant which is deficient in the heptosyltransferase-III (35000hep-) and its parental strain 35000 were analyzed by SDS-PAGE and Western blotting (Figure 2). The LOS from a previously characterized mutant (HD35000R) that synthesizes a highly truncated LOS that lack a terminal LacNAc structure [20] was also analyzed as negative control. The migration pattern of wild-type 35000 (lane 1a) and mutant 35000hep- (lane 3a) appeared to be similar, but 35000 hep - showed one new band with a higher molecular weight. Western blot analysis of this same gel with MAb 3F11, an antibody that recognizes terminal LacNAc, showed immunoreactivity with two prominent bands in the LOS from the mutant strain 35000 hep-, suggesting the presence of LOS containing mono-LacNAc and di-LacNAc glycoforms (lane $3 \mathrm{~b}$ ). Closer inspection revealed that the 35000 wild-type LOS also contained some corresponding higher mass glycoforms, but only as a very minor components (lane $1 \mathrm{~b}$ ).

Mass spectrometric analysis of the $O$-deacylated LOS of the 35000hep- mutant confirmed loss of one core heptose residue compared to the wild-type strain 35000 as expected from the disruption of heptosyltransferaseIII gene. MALDI-MS spectra of O-LOS isolated from strain 35000 hep - grown in liquid broth media (Figure 3a) showed LOS-glycoforms that were shifted down in

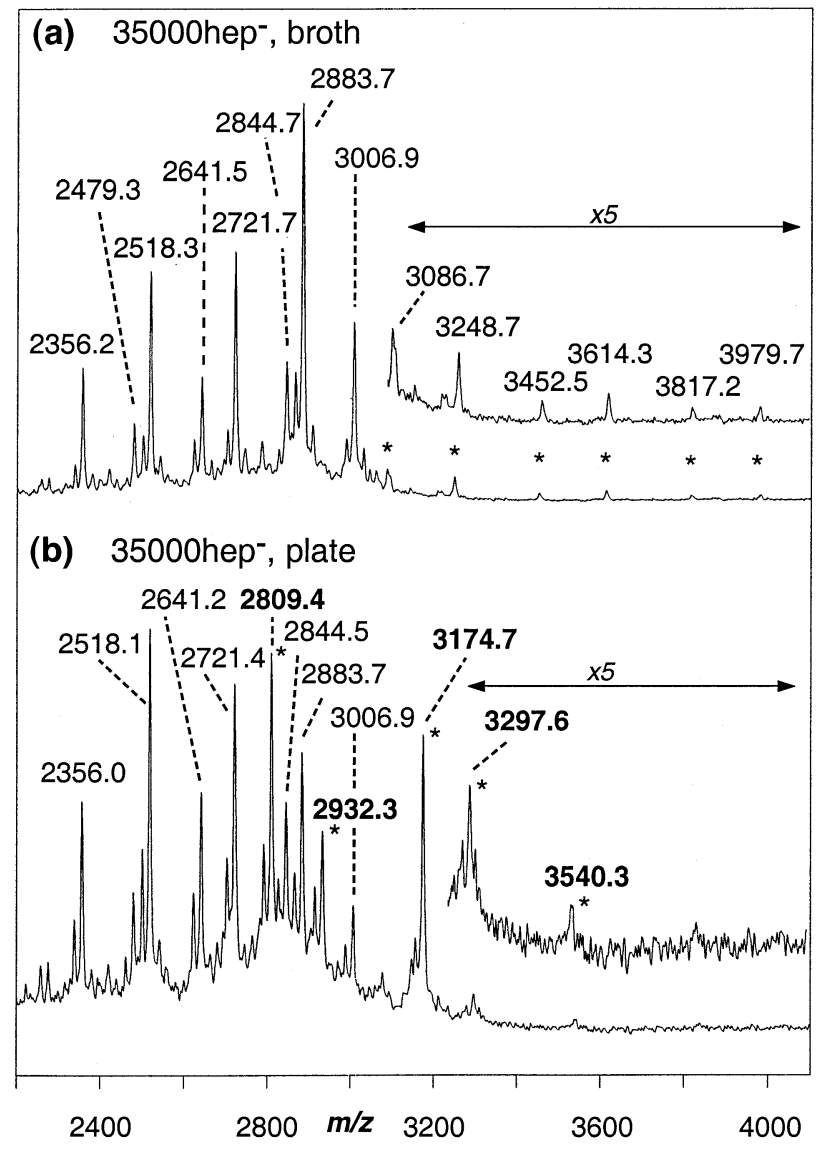

Figure 3. Negative-ion MALDI-TOF mass spectra of O-deacylated LOS from heptosyltransferase knockout mutant strain 35000 hep -. (a) 35000hep - grown in liquid media (broth), and (b) 35000 hep - grown on solid media (plate). Bold labels indicate sialylated species. Spectra were recorded in linear mode yielding average masses.

mass by $192 \mathrm{Da}$, the residue mass for heptose. The deprotonated molecular ion peaks $[\mathrm{M}-\mathrm{H}]^{-}$at $m / z 2518.3$ and 2641.5 correspond to the major wild-type glycoforms terminating in LacNAc, i.e., Gal $\rightarrow$ GlcNAc $\rightarrow$ $\mathrm{Gal} \rightarrow \mathrm{Hep} \rightarrow \mathrm{Glc} \rightarrow \mathrm{Hep}_{2} \rightarrow \mathrm{KdoP}(\mathrm{PEA})_{0,1}-\mathrm{O}$-deacylLipid A, which is now lacking the terminal core heptose-III residue (referred to as $\mathrm{A}_{5}^{\prime}$ according to the nomenclature by Bozue et al. [13]). The amount of PEA substituted on LOS is variable, as evident from the expected mass shifts of $123 \mathrm{Da}$. The peaks at $\mathrm{m} / \mathrm{z} 2356.2$ and $2479.3\left(\mathrm{~A}_{4}^{\prime}\right)$ appear to arise from the additional loss of the terminal galactose, a minor branch structure also seen in the wild-type LOS structure (data not shown). However, in addition to these expected LOS-glycoforms, several new high mass LOS species were observed that suggested other modifications to these LOS in addition to the loss of heptose. For example, LOS species terminating in LacNAc did not appear to be sialylated, unlike the parental wild-type strain in which this is the second most abundant glycoform, i.e., NeuAc $\alpha 2 \rightarrow 3 \mathrm{Gal} \beta 1 \rightarrow 4$ GlcNAc-R $[10,11]$. Rather, several high mass LOS-glycoforms were present whose masses were consistent with the extension of a second 


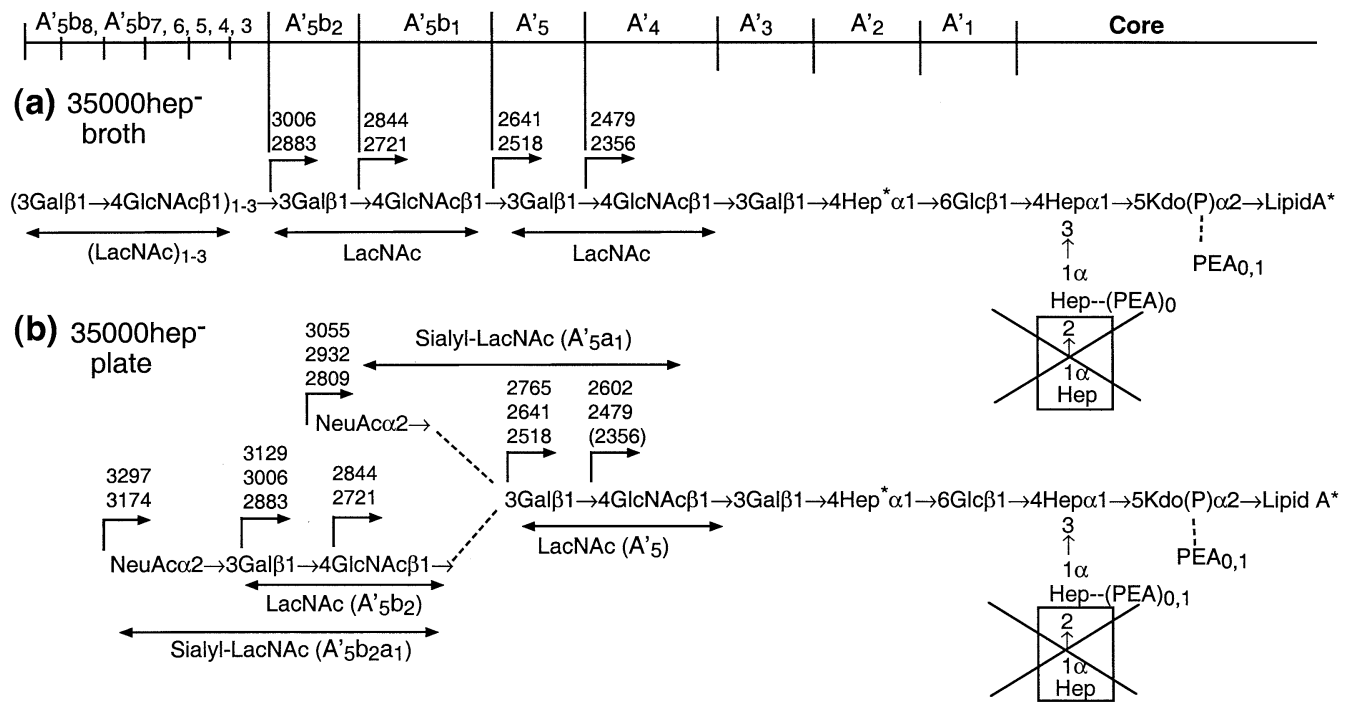

Figure 4. Structures and average masses $[\mathrm{M}-\mathrm{H}]$ for the various $\mathrm{O}$-deacylated LOS-glycoforms from $35000 \mathrm{hep}-$ mutants grown on (a) broth and (b) plates. Nomenclature for LOS-glycoform structure is based on the nomenclature of Bozue et al. [13], for H. ducreyi LOS-glycoforms, where $\mathrm{A}_{n}^{\prime}$ refer to the major oligosaccharide branch structures substituted on Hep-I of the conserved Hep ${ }_{2}$ Kdo-Lipid A* core that lacks Hep-III. The normal position of the missing Hep-III from the LOS obtained from 35000hepis drawn in a crossed out box. All core heptoses are L-glycero-D-manno-heptose with the exception of the branch heptose (labeled with an asterisk) which is D-glycero-D-manno-heptose. Lipid $\mathrm{A}^{*}$ refers to the di-N-acyl-diphosphoryl form generated after hydrazine treatment.

LacNAc moiety at $m / z 2883.7$ and $3006.9\left(\mathrm{~A}_{5}^{\prime} \mathrm{b}_{2}\right.$, mass difference is variable PEA substitution). Further additions of $\mathrm{N}$-acetylhexosamine (i.e., GlcNAc) and hexose (i.e., Gal) to this abundant di-LacNAc structure can be seen as leading to the formation of relatively minor LOS-glycoforms containing as many as five LacNAc repeats, i.e, $\mathrm{m} / \mathrm{z}$ 3086.7, 3248.7, 3452.5, 3614.3, 3817.2, $3979.7\left(\mathrm{~A}_{5}^{\prime} \mathrm{b}_{3}, \ldots, \mathrm{A}_{5}^{\prime} \mathrm{b}_{8}\right.$, respectively). If these higher mass glycoforms contain linear repeats of LacNAc, LOS structures can be depicted as shown in Figure 4, where the mass values listed are those calculated for the average masses and are in agreement with those observed in the corresponding MALDI-MS spectra (Figure $3 a)$. The average molecular masses of the $O$-deacylated LOS-glycoforms are also summarized in Table 1. Although the LOS containing the terminal di-LacNAc was detectable on SDS-PAGE (Figure 2, lane 3), the glycoforms containing polyLacNAc were only visible in gels that were severely overloaded (data not shown).

Unexpectedly, bacteria of the same mutant strain 35000hep - grown on plates (i.e., solid media) expressed a somewhat different set of high mass LOS-glycoforms as can be seen in the corresponding MALDI-MS spectrum (see Figure 3b). As mentioned above, mutant organisms grown in broth expressed glycoforms containing mono- $\left(\mathrm{A}_{5}^{\prime}\right)$ and di-LacNAc $\left(\mathrm{A}_{5}^{\prime} \mathrm{b}_{2}\right)$, as well as less abundant LOS containing polyLacNAc extensions $\left(A_{5}^{\prime} b_{3}, \ldots, A_{5}^{\prime} b_{8}\right)$. In contrast, when this same organism is grown on solid media, a series of major LOS-glycoforms terminating in either a single $\operatorname{LacNAc}\left(\mathrm{A}_{5}^{\prime}, \mathrm{m} / \mathrm{z}\right.$ at 2518.1 and 2641.2) or two LacNAc repeats $\left(\mathrm{A}_{5}{ }_{5} \mathrm{~b}_{2}, \mathrm{~m} / \mathrm{z}\right.$ at 2883.7 and 3006.9) were observed. Moreover, both the mono- and di-LacNAc LOS-glycoforms were partially sialylated, producing structures terminating in NeuAc $\alpha 2 \rightarrow 3 \mathrm{Gal} \beta 1 \rightarrow 4$ GlcNAc $\left(\mathrm{A}_{5}^{\prime} \mathrm{a}_{1}, \mathrm{~m} / z\right.$ at 2809.4 and 2932.3) and in NeuAc $\alpha 2 \rightarrow 3(\mathrm{Gal} \beta 1 \rightarrow 4 \mathrm{GlcNAc})_{2}$ $\left(\mathrm{A}_{5}^{\prime} \mathrm{b}_{2} \mathrm{a}_{1}, \mathrm{~m} / \mathrm{z}\right.$ at 3174.7 and 3297.2$)$, but with no further extensions (these structures are also depicted in Fig-

Table 1. Molecular masses and proposed compositions of O-deacylated LOS from H. ducreyi strain 35000hep-

\begin{tabular}{lccc}
\hline $\begin{array}{l}\text { (LacNAc) }{ }_{n}-R^{\mathrm{a}} \\
n=\end{array}$ & $M_{r}$ (obs. $^{\mathrm{b}}$ & $M_{r}$ (calc. $^{\mathrm{c}}$ & $\Delta M(\mathrm{ppm})$ \\
\hline \hline $0+$ GlcNAc & 2357.16 & 2357.14 & 9 \\
$0+$ GlcNAc & 2480.26 & 2480.19 & 28 \\
1 & 2519.31 & 2519.28 & 12 \\
1 & 2642.46 & 2642.33 & 49 \\
$1+$ GlcNAc & 2722.66 & 2722.48 & 12 \\
$1+$ GlcNAc & 2845.66 & 2845.52 & 49 \\
2 & 2884.71 & 2884.62 & 31 \\
2 & 3007.89 & 3007.66 & 76 \\
$2+$ GlcNAc & 3087.67 & 3087.81 & -45 \\
3 & 3249.74 & 3249.96 & -68 \\
$3+$ GlcNAc & 3453.51 & 3453.15 & 104 \\
4 & 3615.33 & 3615.29 & 11 \\
$4+$ GlcNAc & 3818.23 & 3818.49 & -68 \\
5 & 3980.66 & 3980.63 & 8 \\
$1+$ NeuAc & 2810.39 & 2810.54 & -53 \\
$1+$ NeuAc & 2933.35 & 2933.59 & -82 \\
$2+$ NeuAc & 3175.69 & 3175.88 & -60 \\
$2+$ NeuAc & 3298.62 & 3298.93 & -94 \\
\hline
\end{tabular}

aNumber $n$ refers to repeating LacNAc moieties substituted on the remaining LOS structure, i.e., $\mathrm{R}=\mathrm{Gal}-\mathrm{Hep}-\mathrm{Glc}-\mathrm{Hep}_{2}(\mathrm{PEA})_{0-1}-\mathrm{Kdo}_{\text {-Lipid }}$ $A^{*}$, where Lipid $A^{*}$ is the di-N-acyl-diphosphoryl form

${ }^{b}$ All experimental data was obtained by negative-ion MALDI-MS yielding $[\mathrm{M}-\mathrm{H}]^{-}$ions (average masses) as shown in Figure 2.

${ }^{\mathrm{c} A l l}$ calculated masses are expressed as average masses. 

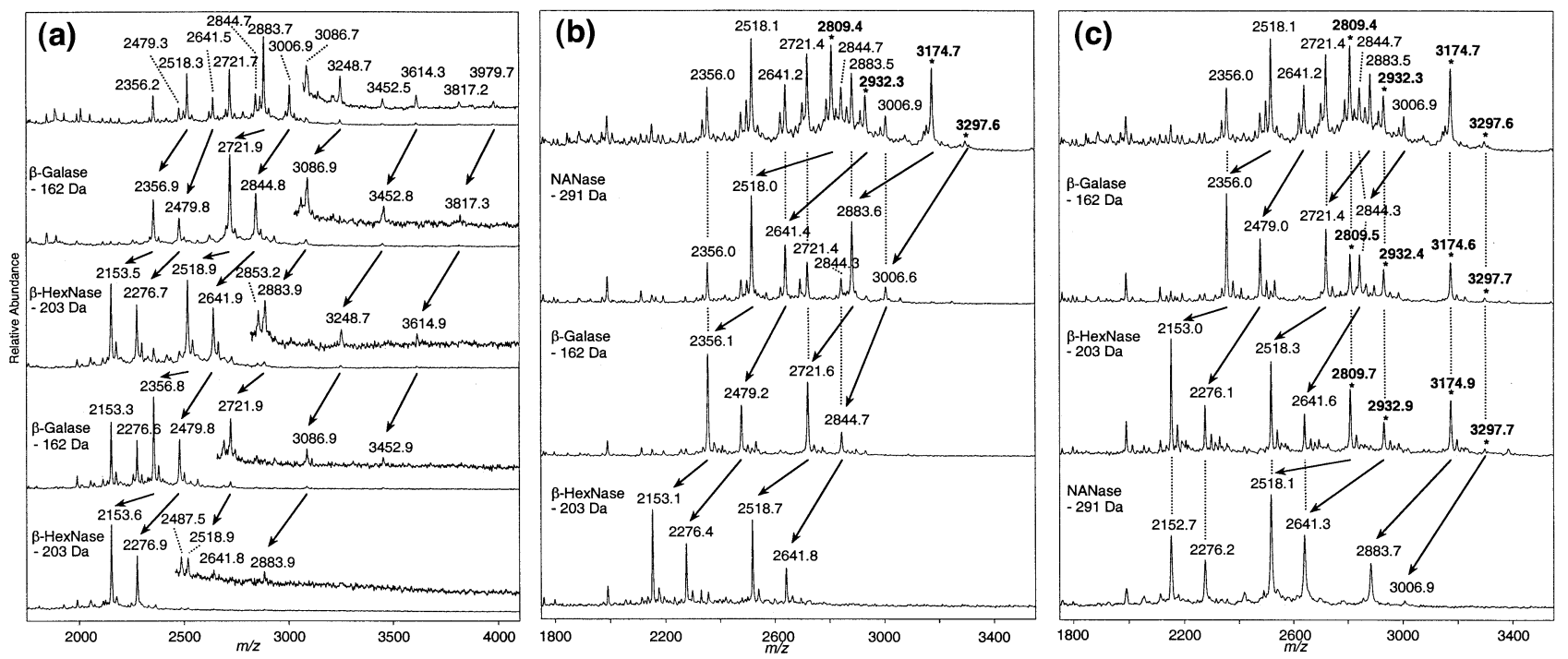

Figure 5. Negative-ion MALDI-TOF mass spectra of O-deacylated LOS mutant strain 35000hepbefore and after exogycosidase digestion as follows: (a) Bacteria grown in broth. From top to bottom, $O$-LOS untreated and then treated in succession with $\beta$-galactosidase, $\beta$ - $\mathrm{N}$-acetylhexosaminidase, $\beta$-galactosidase, and $\beta$-N-acetylhexosaminidase; (b) Bacteria grown on plates. From top to bottom, $\mathrm{O}$-LOS untreated and treated in succession with neuraminidase, $\beta$-galactosidase, and $\beta$ - $\mathrm{N}$-acetylhexosaminidase; (c) Bacteria grown on plates. From top to bottom, O-LOS untreated and treated in succession with $\beta$-galactosidase, $\beta$-N-acetylhexosaminidase, and neuraminidase. Spectra were recorded in linear mode yielding average masses.

ure 4 and their corresponding compositions listed in Table 1).

To better characterize these novel high molecular weight structures (i.e., polyLacNAc LOS), a series of stepwise enzymatic digestions were employed using various combinations of $\beta$-galactosidase, $\beta$ - $\mathrm{N}$-acetylhexosaminidase, and neuraminidase. In one experimental regimen, the original LOS mixture obtained from organisms grown in broth was first treated with $\beta$-galactosidase, followed by successive treatments of $\beta$-Nacetylhexosaminidase, $\beta$-galactosidase, and $\beta$ - $\mathrm{N}$-acetylhexosaminidase (see Figure $5 \mathrm{a}$ ). In this experiment, one expects a downward shift in mass in increments of 162 $\mathrm{Da}$ for loss of terminal $\beta$-linked galactose and $203 \mathrm{Da}$ for loss of terminal $\beta$-linked HexNAc (GlcNAc). Although these high mass LOS-glycoforms were of relative low abundances, one can clearly see the appearance (and/or disappearance) of various molecular ions that can be interpreted as the successive losses of one sugar (Gal or HexNAc) per digestion step for the majority of LOSglycoforms. These mass shifts are consistent with a linear polyLacNAc structure as originally suggested by the immunochemical data (Figure 2).

To examine LOS containing sialic acid (Figure $5 \mathrm{~b}$ and c), neuraminidase was added to the exoglycosidase treatment regimen. As discussed above, there was evidence that the mutant strain 35000hep- grown on plates synthesized abundant sialyl-LacNAc and sialyldi-LacNAc LOS species. To test this hypothesis, O-LOS prepared from plate-grown organisms was sequentially incubated with neuraminidase $(\Delta m=291 \mathrm{Da}), \beta$-galactosidase and $\beta$-N-acetylhexosaminidase (Figure $5 \mathrm{~b}$ ). Al- ternatively, these same O-LOS were treated with $\beta$-galactosidase, followed by $\beta$-N-acetylhexosaminidase and then neuraminidase (Figure $5 \mathrm{c}$ ). Molecular ions at $\mathrm{m} / \mathrm{z}$ 2809.4/2932.3 $\left(\mathrm{A}_{5}^{\prime} \mathrm{a}_{1}\right)$ and at $\mathrm{m} / \mathrm{z}$ at $3174.7 / 3297.2$ $\left(A_{5}^{\prime} b_{2} a_{1}\right)$ were easily identified as sialylated glycoforms by their susceptibility to neuraminidase treatment, but they were resistant to prior treatment with $\beta$-galactosidase or $\mathrm{N}$-acetylhexosaminidase. In addition, these data are consistant with LOS structures where LacNAc serves as the acceptor for sialic acid.

To confirm that these high molecular weight LOSglycoforms containing polyLacNAc and sialylated LacNAc structures emanate from a single oligosaccharide branch, series of tandem mass spectrometry were performed on various LOS preparations. MALDI postsource decay (PSD) and ESI tandem mass spectrometry (ESI-MS/MS) were used to establish the overall architecture and branching pattern of these structures. Using MALDI-PSD, several molecular ions were individually selected and their metastable decomposition fragments were detected by the reflectron mass analyzer. As shown in Figure 6, MALDI-PSD spectra from O-deacylated LOS species with $[\mathrm{M}-\mathrm{H}]^{-}$at $m / z 3006.7$ (Figure 6a), $m / z 2883.7$ (Figure $6 b$ ), and $m / z 2721.7$ (Figure 6c) were recorded in the negative-ion mode and provided limited structural information on the oligosaccharide (and Lipid A) portion of the LOS. For example, at high mass the MALDI-PSD spectrum of the $O-\mathrm{LOS}$ at $\mathrm{m} / \mathrm{z}$ 3006.7 showed loss of PEA and $\beta$-elimination of phosphoric acid $\left(-\mathrm{H}_{3} \mathrm{PO}_{4}, \Delta \mathrm{m}=-98 \mathrm{Da}\right)$ whereas the MALDI-PSD spectra of $O$-LOS at $\mathrm{m} / \mathrm{z} 2883.7$ and 2721.7 showed a loss of phosphate only. At lower masses, 


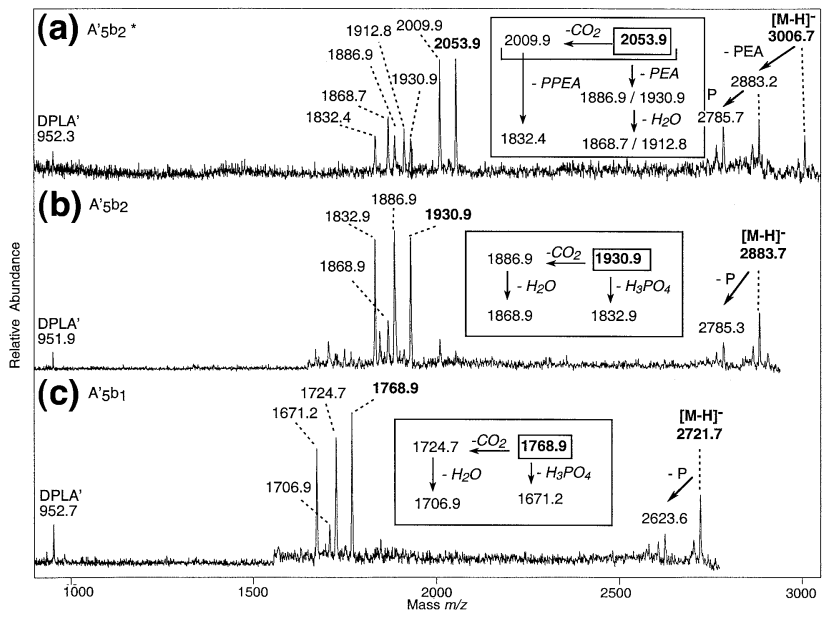

Figure 6. Negative-ion MALDI-PSD spectrum of O-deacylated LOS selecting deprotonated precursor ion (a) $[\mathrm{M}-\mathrm{H}]^{-}$at $\mathrm{m} / \mathrm{z}$ $3006.7\left(\mathrm{~A}_{5}^{\prime} \mathrm{b}_{2}{ }^{*}\right.$, with 1 PEA and 2 LacNAc); (b) $[\mathrm{M}-\mathrm{H}]^{-}$at $\mathrm{m} / \mathrm{z}$ $2883.7\left(\mathrm{~A}_{5}^{\prime} \mathrm{b}_{2}\right.$, with 0 PEA and di-LacNAc structure), and (c) $[\mathrm{M}-$ $\mathrm{H}]^{-}$at $m / z 2721.7\left(\mathrm{~A}_{5}^{\prime} \mathrm{b}_{1}\right.$, with 0 PEA and terminal GlcNAc-GalGlcNAc structure).

fragments are seen for the corresponding di-N-acyl diphosphoryl Lipid A ( $m / z$ 952) and oligosaccharide moieties by cleavage of the labile Kdo-O-Lipid A glycosidic bond [9]. The oligosaccharide moieties undergo further fragmentation including the loss of $\mathrm{CO}_{2}$ from Kdo (-44 Da), PEA (-123 Da), phosphoric acid (-98 $\mathrm{Da})$, and $\mathrm{H}_{2} \mathrm{O}(-18 \mathrm{Da})$. The molecular ions at $\mathrm{m} / \mathrm{z}$ 3006.7 and 2883.7 were both assigned as di-LacNAc containing glycoforms $\left(\mathrm{A}_{5}^{\prime} \mathrm{b}_{2}\right)$ containing either one or no PEA. The major oligosaccharide fragments at $\mathrm{m} / \mathrm{z}$ 2053.9/2009.9 (Figure 6a) and $\mathrm{m} / \mathrm{z}$ 1930.9/1886.9 (Figure 6b) differed by $123 \mathrm{Da}$, confirming that PEA heterogeneity is present on the oligosaccharide rather than the Lipid A moiety. The MALDI-PSD spectrum of molecular ion at $\mathrm{m} / \mathrm{z} 2721.7\left(\mathrm{~A}_{5}^{\prime} \mathrm{b}_{1}\right)$ yielded oligosaccharide fragments at $m / z 1768.9$ and 1724.7 that can be assigned as oligosaccharide moiety containing no PEA and lacking a single galactose extension from a terminal monoLacNAc species (1930.9-162 Da). However, no fragmentation was observed in these PSD spectra that revealed sequence information of the oligosaccharide.

To obtain more detailed information of the oligosaccharide moiety, the LOS-glycoforms were cleaved by acid treatment and separated from the Lipid A by centrifugation and size exclusion chromatography. It should be noted that hydrolysis of LOS also results in the $\beta$-elimination of phosphate and phosphorylphosphoethanolamine (PPEA) from Kdo forming anhydroKdo at the reducing terminus [10, 32]. The exact masses of the released oligosaccharides with up to five lactosamine units were determined by ESI-MS in positive ionization mode and are summarized in Table 2. For most oligosaccharides, mass accuracies $\leq 20 \mathrm{ppm}$ could be achieved using the previously well characterized glycoforms $\left(\mathrm{A}_{5}^{\prime}\right.$ and $\left.\mathrm{A}_{5} \mathrm{~b}_{1}\right)$ as internal standards; oligosaccharides containing four and five LacNAc units were
Table 2. Molecular masses of oligosaccharides containing 1 to 5 LacNAc units prepared from $H$. ducreyi strain 3500hep-

\begin{tabular}{lccc}
\hline \# LacNAc $^{\text {a }}$ & $M_{r}$ (obs.) $^{\text {b }}$ & $M_{r}$ (calc.) & $\Delta M[\mathrm{ppm}]$ \\
\hline \hline 1 & 1485.493 & 1485.486 & 4 \\
$1+$ GlcNAc & 1688.544 & 1688.566 & -13 \\
2 & 1850.616 & 1850.619 & -1 \\
$2+$ GlcNAc & 2053.704 & 2053.698 & -3 \\
3 & 2215.709 & 2215.751 & 19 \\
$3+$ GlcNAc & 2418.816 & 2418.830 & 6 \\
4 & 2580.776 & 2580.883 & 41 \\
$4+$ GlcNAc & $n . d$. & - & - \\
5 & 2945.860 & 2946.015 & 53 \\
\hline
\end{tabular}

aNumbers refer to repeating LacNAc moieties substituted on the conserved Gal-Hep-Glc-Hep ${ }_{2}(\mathrm{PEA})_{0-1}$-anhydroKdo oligosaccharide portion of the LOS. At the reducing terminus, anhydroKdo oligosaccharide portion of the LOS. At the reducing terminus, anhydroKdo is formed by $\beta$-elimination of phosphate (P) or pyrophospho-ethanolamine (PPEA) under acid conditions [9].

${ }^{\mathrm{b}}$ All masses were recorded by ESI-MS in positive-ion mode yielding ${ }^{12}[\mathrm{C}]$-containing isotopic masses.

'Previously well characterized glycoforms $A^{\prime}{ }_{5}$ and $A^{\prime}{ }_{5} b_{1}$ were used as internal calibration standards.

nd glycoform was not detected.

externally calibrated. Sialylated LOS that were isolated from bacteria grown on plates had to be hydrolyzed using a milder method to retain the sialic acid residue. The latter oligosaccharides were subsequently analyzed by MALDI-MS in linear, negative-ion mode yielding molecular ion peaks $\left[\mathrm{M}-\mathrm{H}^{-}\right.$at $m / z 1485.3\left(\mathrm{~A}_{5}^{\prime}\right), 1689.0$ $\left(\mathrm{A}_{5}^{\prime} \mathrm{b}_{1}\right), 1850.2\left(\mathrm{~A}_{5}^{\prime} \mathrm{b}_{2}\right)$, and the sialylated species at $\mathrm{m} / \mathrm{z}$ 1776.4 $\left(\mathrm{A}_{5}^{\prime} \mathrm{a}_{1}\right)$ and $2141.6\left(\mathrm{~A}_{5}^{\prime} \mathrm{b}_{2} \mathrm{a}_{1}\right)$.

As previously reported [33,34], MALDI-PSD can be used to obtain sequence information of carbohydrates. MALDI-PSD spectra recorded for two such oligosaccharides as shown in Figure 7 where the sodium adducts $[\mathrm{M}+\mathrm{Na}]^{+}$at $m / z 1874.0$ and 2604.8 contain either two or four terminal LacNAc structures, respectively. For the di-LacNAc oligosaccharide, a series of sequenceions was generated that correspond to cleavage of the carbohydrate chain, including a Y-ion $(\mathrm{m} / \mathrm{z}$ 1509.2, 1346.9, 1143.7, and 981.3), B-ion $(\mathrm{m} / \mathrm{z} 1653.9,1461.8$, $1269.4,1107.9,915.9,753.5$, and 388.6) and corresponding C-ion series (see inset, Figure 7a). The most abundant fragment-ion at $m / z 1653.9$ was assigned to a $B_{8}$-ion as a simple loss of Kdo from the reducing terminus. A few fragment-ions were observed that result from two or more cleavages (internal fragments), such as further loss of Kdo from $\mathrm{Y}_{5 \alpha}$-ion $(\mathrm{m} / \mathrm{z}$ 923.8, internal fragment). In addition, a MALDI-PSD spectrum of a polyLacNAccontaining oligosaccharide with four repeats was recorded (Figure $7 \mathrm{~b}$ ). In the original unseparated O-LOS mixture from which this oligosaccharide was obtained, the corresponding glycoform was only observed as a minor component (i.e., O-deacylated LOS at $\mathrm{m} / \mathrm{z}$ 3614.3, Figure 3a). Despite its weak signal and the large decrease in the abundance of fragments, the MALDI-PSD spectrum yielded B-type fragment ions at $\mathrm{m} / \mathrm{z}$ 388.0, $550.2,753.7,1118.3,1484.0,1645.8$, and 1837.7 that were consistent with a linear (LacNAc) $)_{4}$ structure as shown in Figure 6c. 

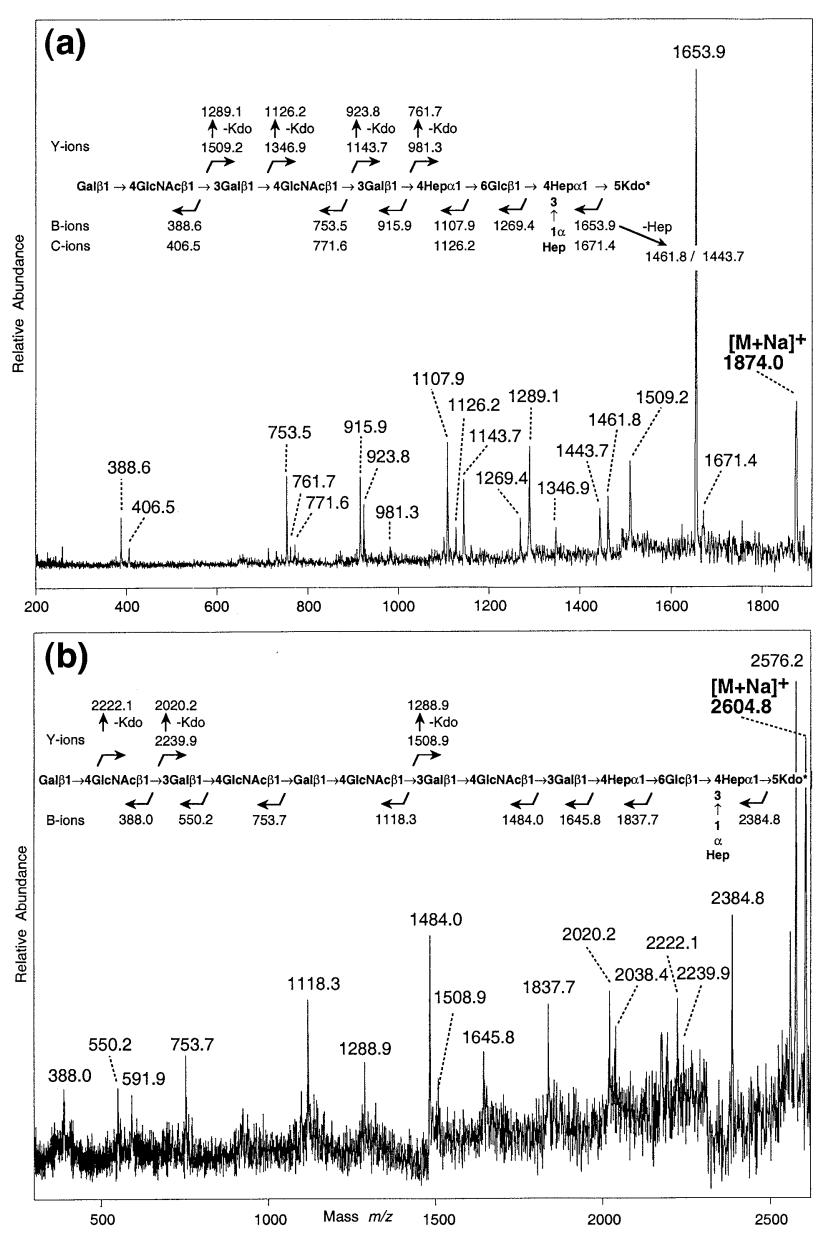

Figure 7. Positive-ion MALDI-PSD spectrum of polyLacNAc containing oligosaccharides. Precursors ions selected were (a) $[\mathrm{M}+\mathrm{Na}]^{+}$at $m / z 1874.0$ (di-LacNAc) and (b) $[\mathrm{M}+\mathrm{Na}]^{+}$at $\mathrm{m} / \mathrm{z}$ 2604.8 (tetra-LacNAc). Kdo* refers to anhydroKdo. Ion nomenclature is according to that proposed by Domon and Costello [40].

MALDI-PSD characterization of these LOS species, either as free oligosaccharides or O-LOS, became problematic as one progressed to higher mass or to LOSglycoforms that contained sialic acid. To see if ESI MS/MS might yield more complete sequences for these glycoforms, we investigated both O-LOS and free oligosaccharides (in mixtures) using a high performance quadrupole time-of-flight instrument. Under ESI conditions, free oligosaccharides form both doubly and triply charged molecular ions. For some oligosaccharides, only molecular ion species containing one or more cations were observed, e.g., $[\mathrm{M}+2 \mathrm{H}+\mathrm{Na}]^{3+}$ and $[\mathrm{M}+2 \mathrm{H}+\mathrm{K}]^{3+}$. Metal ions such as $\mathrm{Na}^{+}$and $\mathrm{K}^{+}$are likely introduced into the samples from the coated nanoelectrospray needles. However, as has been reported by others [35], oligosaccharides that are partially metalated can sometimes undergo more extensive fragmentation. ESI MS/MS experiments on oligosaccharides containing di- to penta-LacNAc moieties, as well as some sialylated counterparts, were therefore carried out on various cationated molecular ions.

ESI-MS/MS spectra of the oligosaccharide contain-
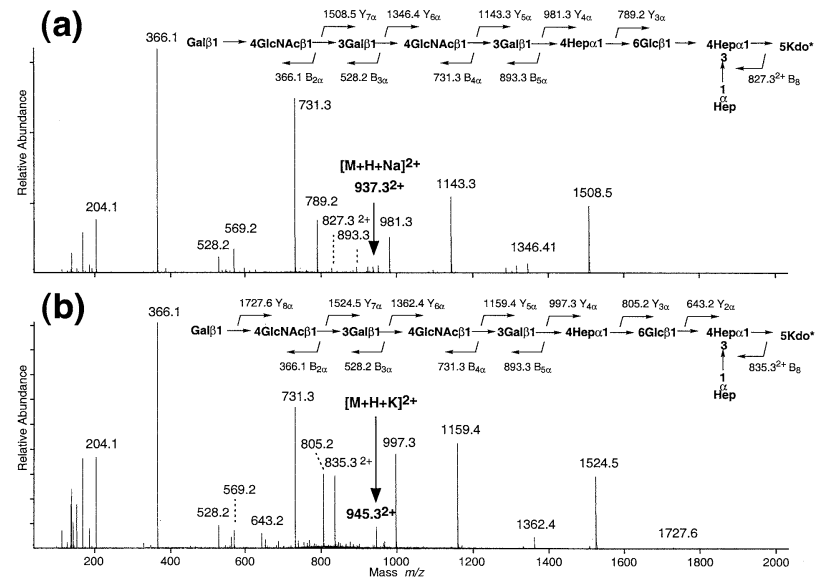

Figure 8. ESI-MS/MS spectrum of doubly charged metalated di-LacNAc containing oligosaccharide (a) $[\mathrm{M}+\mathrm{H}+\mathrm{Na}]^{2+}$ at $\mathrm{m} / \mathrm{z}$ $937.3^{2+}(\mathrm{M}=1850.6 \mathrm{Da})$ and $(\mathbf{b})[\mathrm{M}+\mathrm{H}+\mathrm{K}]^{2+}$ at $\mathrm{m} / \mathrm{z} 945.3^{2+}$ $(\mathrm{M}=1850.6 \mathrm{Da})$. B-type fragment-ions appear as protonated species whereas Y-type ions appear as metalated species. Kdo* refers to anhydroKdo.

ing di-LacNAc $\left(\mathrm{A}_{5}^{\prime} \mathrm{b}_{2}, \mathrm{M}=1850.6\right)$ were obtained by selecting a doubly charged sodiated precursor ion, $[\mathrm{M}+\mathrm{H}+\mathrm{Na}]^{2+}$ at $m / z 937.3^{2+}$ as well as the doubly charged potassium precursor ion, $[\mathrm{M}+\mathrm{H}+\mathrm{K}]^{2+}$ at $m / z$ $945.3^{2+}$ (Figure 8). Most fragment-ions were singly charged $\mathrm{Y}$ - and B-ion fragments and were analogous to those observed in the MALDI-PSD spectrum (see Figure 7), but more complete. B-ions appeared as protonated ions whereas the corresponding Y-ions contained sodium and potassium adducts. The presence of $\mathrm{Na}^{+}$or $\mathrm{K}^{+}$in the Y-ions was almost certainly due to the presence of a carboxyl group in the reducing terminal Kdo moiety. Direct comparison of ESI-MS/MS spectra from partially sodiated and potassiated doubly charged precursor ions of the same species allowed us to easily differentiate between B- and Y-ions. Protonated B-ions showed identical masses in both spectra, such as at $\mathrm{m} / \mathrm{z}$ $366.1\left(\mathrm{~B}_{2 \alpha}\right)$ and $731.3\left(\mathrm{~B}_{4 \alpha}\right)$. In contrast, singly charged metalated Y-ions revealed a mass difference of $16 \mathrm{Da}$, corresponding to the mass difference between $\mathrm{K}^{+}$and $\mathrm{Na}^{+}$, i.e., $Y_{5 \alpha}$ and $Y_{7 \alpha}$ were observed at $m / z 1143.3$ and $\mathrm{m} / \mathrm{z} 1508.5$ as sodiated ion and at $\mathrm{m} / \mathrm{z} 1159.4$ and 1524.5 as potassiated ions.

ESI-MS/MS proved especially useful sequencing high molecular weight oligosaccharides. In one experiment, a triply charged precursor ion with $[\mathrm{M}+2 \mathrm{H}+$ $\mathrm{Na}]^{3+}$ at $m / z 814.61^{3+}$ was selected that corresponds to an oligosaccharide species containing $(\mathrm{LacNAc})_{3}$ plus an additional GalNAc ( $\mathrm{A}_{5}^{\prime} \mathrm{b}_{5}, \mathrm{M}=2418.82 \mathrm{Da}$ ) (data not shown). An abundant series of B-ion fragments was present that was complete from $B_{1 \alpha}$ through $B_{6 \alpha}(\mathrm{m} / z$ 204.1, 366.2, 569.2, 731.3, 934.4 and 1096.4), encompassing what is clearly a linear polyLacNAc structure. The carbohydrate molecule with the highest molecular weight was detected for the $[\mathrm{M}+3 \mathrm{H}]^{3+}$ at $\mathrm{m} / \mathrm{z} 982.96^{3+}$ $\left(\mathrm{M}=2945.86, \mathrm{~A}_{5}^{\prime} \mathrm{b}_{8}\right)$. The ESI-MS spectrum of the original oligosaccharide fraction is shown in Figure 9 


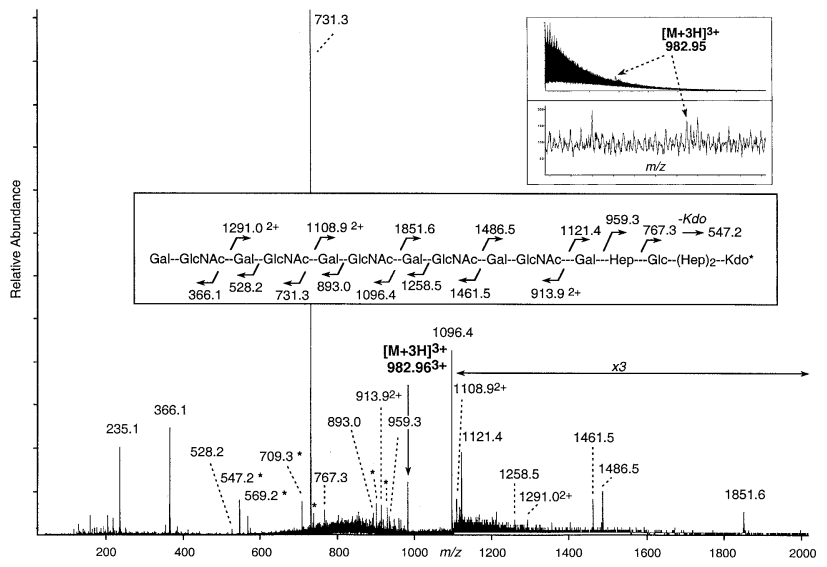

Figure 9. ESI-MS/MS spectrum of 5 LacNAc unit containing oligosaccharide $\mathrm{A}_{5}^{\prime} \mathrm{b}_{8}$. Selection of triply charged, protonated precursor ion $[\mathrm{M}+3 \mathrm{H}]^{3+}$ at $\mathrm{m} / z 982.96^{3+}(\mathrm{M}=2945.86)$. Kdo* refers to anhydroKdo. Ions marked with an asterisk are internal ions.

(inset) and revealed a very weak signal at $\mathrm{m} / \mathrm{z} 982.96$ $(z=3)$. ESI-MS/MS analysis of this isolated oligosaccharide generated numerous $\mathrm{Y}$ - and B-ion fragments that were consistent with a linear $(\mathrm{LacNAc})_{5}$ terminal structure. The most prominent peak of the spectrum at $m / z 731.3$ was assigned as $B_{4 \alpha}$-ion (terminal di-LacNAc). The corresponding counter-ion $\mathrm{Y}_{11 \alpha^{-}}$-ion at $\mathrm{m} / \mathrm{z} 1108.9^{2+}$ $(\mathrm{M}=2216.7)$ was also observed. Several other of these ion-pairs helped to confirm the (LacNAc) $)_{5}$ structure, including the $\mathrm{B} / \mathrm{Y}$-ion pairs at $m / z 366.1 / 1291.0^{2+}\left[\mathrm{B}_{2 \alpha} /\right.$ $\left.\mathrm{Y}_{13 \alpha}^{2+}\right], m / z$ 1096.4/1851.6 [B $\left.{ }_{6 \alpha} / \mathrm{Y}_{9 \alpha}\right], m / z$ 1461.5/1486.5 $\left[\mathrm{B}_{8 \alpha} / \mathrm{Y}_{7 \alpha}\right]$, and $m / z$ 913.9 $9^{2+} / 1121.4\left[\mathrm{~B}_{10 \alpha}^{2+} / \mathrm{Y}_{5 \alpha}\right]$. The B-ion components of these ion pairs are formed by fragmentation of GlcNAc-Gal glycosidic bonds and are presumably stabilized by the nitrogen in the GlcNAc residue.

Although ESI-MS/MS spectra provided complete sequences of the terminal LacNAc regions of all oligosaccharides that were isolated, a different approach was needed to obtain sequence data on oligosaccharide branches from low abundant LOS-glycoforms and/or ones that contain sialic acid. In these cases, isolation of the corresponding oligosaccharides was difficult, especially for those that contain sialic acid. Although negative-ion ESI-MS/MS of the O-LOS mixtures can yield useful data, it is our experience that positive-ion ESIMS/MS of dephosphorylated O-LOS yields more abundant molecular ions and more sequence information. To obtain LOS-glycoforms that are more suitable to ESIMS/MS under positive-ion conditions, the O-deacylated LOS glycoforms were treated with aqueous HF $\left(4{ }^{\circ} \mathrm{C}, 12 \mathrm{~h}\right)$ to remove PEA and phosphate. HF-treatment of the O-LOS species at $\mathrm{M}_{\text {calc }}=2721 / 2744 \mathrm{Da}$ $\left(\mathrm{A}_{5}{ }_{5} \mathrm{~b}_{1}\right)$ would be expected to cleave one phosphate and 0-1 PEA from the Kdo residue, and two phosphate groups from the Lipid A moiety to yield a dephosphorylated O-LOS glycoform with an exact mass of $\mathrm{M}_{\text {calc }}=$ 2481.0894. The corresponding tandem mass spectrum of this glycoform was obtained by selecting the doubly charged precursor ion at $[\mathrm{M}+2 \mathrm{H}]^{2+}=\mathrm{m} / \mathrm{z} 1241.531$
$\left(\mathrm{M}_{\mathrm{obs}}=2481.047, \Delta \mathrm{m}=17 \mathrm{ppm}\right.$ ); (spectrum not shown). Although the Lipid A moiety is still present, most fragment ions present in the MS/MS spectrum were B- and/or Y-ions originating from the oligosaccharide portion including an abundant B-ion $\left(\mathrm{B}_{1 \alpha-4 \alpha}\right.$ at $m / z 204.1,366.1,569.2,731.3)$ and $Y$-ion series $\left(Y_{3 \alpha-7 \alpha}\right.$ at $m / z 2279.3,2116.9,1913.9,1751.7$, and 1559.8). Together, these ions clearly defined the non-reducing terminal sequence GlcNAc $\rightarrow$ Gal $\rightarrow$ GlcNAc $\rightarrow$ Gal $\rightarrow$ Hep. This approach, therefore, provided sufficient sensitivity and efficiency to sequence an intact LOS without chemically cleaving and separating the resulting oligosaccharide from the Lipid A prior to mass spectrometric analysis.

To test the sensitivity of the ESI-MS/MS approach for sequencing even larger and more labile O-LOS species, we examined two sialylated glycoforms. As mentioned earlier, when the $H$. ducreyi 35000hepstrain is grown on solid media, LOS terminating in sialyl-LacNAc $\left(\mathrm{A}_{5}^{\prime} \mathrm{a}_{1}\right)$ and in sialyl-di-LacNAc $\left(\mathrm{A}_{5}^{\prime} \mathrm{b}_{2} \mathrm{a}_{1}\right)$, are the major species formed (Figure $3 \mathrm{~b}$ and 4 ). Tandem mass spectrometry of the HF-treated O-LOS-glycoform containing a single terminal sialyl-LacNAc moiety acid (precursor-ion with $[\mathrm{M}+\mathrm{H}+\mathrm{Na}]^{2+}$ at $m / z$ 1296.530, $\mathrm{M}$ $=2569.061, \Delta \mathrm{m}=17 \mathrm{ppm}$, data not shown) yielded characteristic fragment ions such as $\mathrm{Y}$-ions $\left(\mathrm{Y}_{3 \alpha-7 \alpha}\right)$, and $\mathrm{B}_{1 \alpha}$ and $\mathrm{B}_{3 \alpha}$-ions at $m / z 292.1$ and $657.2\left(\mathrm{~B}_{3 \alpha}-18\right.$ ion at $m / z$ 639.2), which gave structural proof that a terminal sialic acid residue was added to the terminal LacNAc moiety. The presence of the abundant sialylated di-LacNAc species $\left(\mathrm{A}_{5}^{\prime} \mathrm{b}_{2} \mathrm{a}_{1}\right)$ was of considerable interest, as the di-LacNAc species has been reported to be present in wild type strains but not the sialylated counterpart [14]. To obtain unambiguous data for this sialylated diLacNAc glycoform, an ESI-MS/MS spectrum was recorded for the dephosphorylated O-LOS of the [M $+\mathrm{H}$ $+\mathrm{Na}]^{2+}$ at $m / z 1479.082(\mathrm{M}=2934.166, \Delta \mathrm{m}=24 \mathrm{ppm}$, see Figure 10). Consistent with previous data, most fragment-ions originated from the oligosaccharide moiety. In particular, the $\mathrm{B}_{1 \alpha}$ and $\mathrm{B}_{3 \alpha}$-ions at $m / z 292.1$ and $657.3\left(\mathrm{~B}_{3 \alpha}-18\right.$ ion at $\left.m / z 639.2\right)$ proved that sialic acid was substituted on the terminal LacNAc acceptor. An internal di-LacNAc fragment ion at $\mathrm{m} / \mathrm{z} 731.3$ and the characteristic $\mathrm{B}_{5 \alpha}$-ion at $\mathrm{m} / \mathrm{z} \quad 1022.4$ were especially abundant. In addition, fragment-ions at $\mathrm{m} / \mathrm{z} 2164.7$, 1873.6, 1508.5, 1346.4, and 1143.3 were assigned to oligosaccharide fragments that had lost the Lipid A moiety as well as one or terminal sugars (Figure 10, inset).

\section{Conclusions}

In a previous study by our group [20], an H. ducreyi heptosyltransferase-III knockout mutant was constructed from a prototype strain 35000 and reported to synthesize a LOS that contained a series of unexpected high mass glycoforms. Moreover, the masses of these high mass LOS species were consistent with the formation of a series of polyLacNAc and corresponding sialylated structures. At the time, the structural evi- 


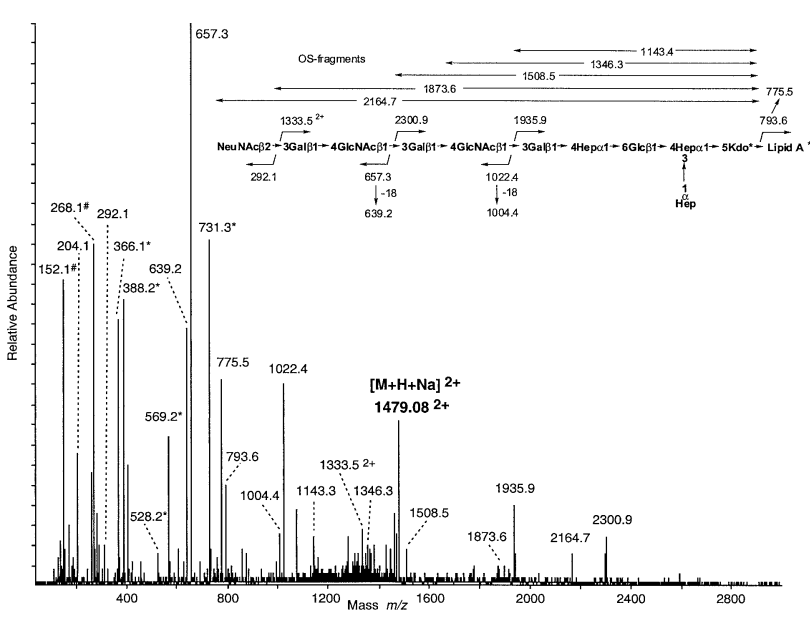

Figure 10. ESI-MS/MS spectrum of HF-treated O-deacylated LOS $\mathrm{A}_{5}^{\prime} \mathrm{b}_{2} \mathrm{a}_{1}$ containing terminal sialyl-(LacNAc) $)_{2}$. Selection of doubly charged, protonated precursor ion with $[\mathrm{M}+\mathrm{H}+\mathrm{Na}]^{2+}$ at $m / z 1479.08(\mathrm{M}=2934.17 \mathrm{Da})$. Kdo* refers to anhydroKdo; Lipid $\mathrm{A}^{*}$ refers to the dephosphorylated di-N-acyl form generated after hydrazine and HF treatment. Ions marked with an asterisk are internal ions; ions marked with a pound sign (\#) are background ions.

dence for these assignment was limited to mass and immunochemical data and no sequence data was presented. In this study, we used a combination of exoglycosidase digestion and mass spectrometric techniques (MALDI-MS, MALDI-PSD, ESI-MS, and ESI-MS/MS) to provide structural proof for these novel glycoforms. In addition, we have evaluated the various MS approaches for their overall suitability to analyze and sequence low-abundance, high-mass glycolipids in complex mixtures.

The combination of exoglycosidase digestion and mass spectrometry were critical to defining these structures. With few exceptions, however, these two techniques have not been used together in the analysis of complex glycan mixtures [36, 37]. In our analysis, we successfully employed up to four consecutive exoglycosidase digestion steps on LOS mixtures containing as many as 15 species, and the mass shifts of the constituent glycoforms could be easily followed to provide information on anomeric centers, sugar types, and linkages. The enzymes $\beta$-galactosidase and $\beta$ - $\mathrm{N}$-acetylhexosaminidase, for example, are both highly specific for $\beta$-anomeric glycosidic bonds. In addition, $\beta$-galactosidase clearly favors hydrolysis of $\beta 1-6$ and $\beta 1-4$ linked Gal-GlcNAc over the corresponding ( $\beta 1-3)$ bonds (relative hydrolysis rates, 100:75:1, respectively). Together with the immunochemical data that indicated the presence of terminal LacNAc epitopes, the precise linkages, sugar types, and anomeric configurations could be readily assigned.

The use of MALDI-MS and ESI-MS for the analysis of bacterial lipooligosaccharides as well as other types of glycans have been well established tools for molecular weight and structure analysis. As we have previously reported [9, 19], MALDI and MALDI-PSD can provide data regarding molecular weight, phosphorylation state, and heterogeneity on the Lipid A and oligosaccharides portions of LOS. However, sequence-specific ions for the oligosaccharide portion of LOS is best obtained from the purified oligosaccharide after mild acid hydrolysis. A core oligosaccharide substituted with up to four repeating LacNAc disaccharides yielded extensive, although somewhat weak, Y- and B-type fragment ions. The tetra-LacNAc glycoform appeared to be near or at the limit of MALDI-PSD sensitivity, as higher masses or glycans substituted with sialic acid did not yield sufficient fragments to assign the structure. In contrast, ESI-MS/MS on a quadrupole o-TOF instrument proved especially efficient and sensitive providing extensive fragments on the least abundant, high-mass OS structures (up to five LacNAc repeats). This capability was critical to obtain sequence information on the unseparated LOS mixtures (after $\mathrm{O}$ deacylation and HF treatment).

The biological significance of these polyLacNAc structures in the LOS of this $H$. ducreyi mutant strain is unclear. This is the first time that a LOS containing a polyLacNAc structure has been identified in bacteria. Regardless of the precise function of these polyLacNAc structures, or even whether they exist in wild-type organisms, our data suggest that a biosynthetic pathway is available to this pathogen that was previously unknown. The observed polyLacNAc and sialylated LOS analogs were present as relatively minor components in a complex glycoform mixture. It is entirely possible, however, that these polyLacNAc structures could be the major forms expressed in the human host. In N. gonorrhoeae, a shift to higher molecular weight glycoforms containing 1-2 terminal LacNAc residues was shown to occur after human challenge [38]. Similar structures, if expressed by $H$. ducreyi under in vivo conditions, would likely play important roles in allowing the organism to survive and/or replicate. The analysis of $H$. ducreyi recovered from the human challenge model may be able to confirm or disprove the presence of polyLacNAc, although such an analysis will be difficult because of the very low number of organisms that are present in these lesions [39]. However, should this polyLacNAc structural phenotype be stable enough to allow for growth in primary culture after human challenge, sufficient material should be obtainable. Our observation that $H$. ducreyi grown in liquid media yields primarily non-sialylated polyLacNAc LOS structures, while those that are grown on plates yield sialylated LOS, may be a critical observation in terms of a possible regulatory control of LOS sialylation. $H$. ducreyi can adhere to a number of human cell types and have been observed as large colonies, and, therefore organisms grown on surfaces (i.e., solid media) may mimic aspects of this in vivo adherent phenotype. Overall, the data present here has provided new insight into possible roles for LOS in the pathobiology of $H$. ducreyi. 


\section{Acknowledgments}

The authors wish to acknowledge the financial support of this work by grants from the National Institutes of Health AI31254 (to BWG) and AI30006 (AAC). Special thanks to Dr. Nancy Phillips for helpful discussions. We also acknowledge Applied Biosystems (Framingham, MA) for the generous support of the MALDI-TOF instrumentation in our laboratory (to BWG).

\section{References}

1. Trees, D. L.; Morse, S. A. Clin. Microbiol. Rev. 1995, 8, 357-375.

2. Jessamine, P. G.; Ronald, A. R. Med. Clin. North Am. 1990, 74, 1417-1431.

3. Spinola, S. M.; Orazi, A.; Arno, J. N.; Fortney, K.; Kotylo, P.; Chen, C. Y.; Campagnari, A. A.; Hood, A. F. J. Infect. Dis. 1996, 173, 394-402.

4. Centers for Disease Control and Prevention. Morbid. Mortal. Weekly Rep 1998, 47, 1-24.

5. Ison, C. A.; Dillon, J. A.; Tapsall, J. W. . Lancet 1998, 351(Suppl. 3), $8-11$.

6. Abeck, D.; Korting, H. C. Acta Dermato. Venereol. Suppl. 1991, 174, 5-20.

7. Alfa, M. J.; DeGagne, P. Microb. Pathog. 1997, 22, 39-46.

8. Gibson, B. W.; Campagnari, A. A.; Melaugh, W.; Phillips, N. J.; Apicella, M. A.; Grass, S.; Wang, J.; Palmer, K.; Munson, R. S. J. J. Bacteriol. 1997, 179, 5062-5071.

9. Gibson, B. W.; Engstrom, J. J.; John, C. M.; Hines, W.; Falick, A. M. J. Am. Soc. Mass Spectrom. 1997, 8, 645-658.

10. Melaugh, W.; Phillips, N. J.; Campagnari, A. A.; Tullius, M. V.; Gibson, B. W. Biochemistry 1994, 33, 13070-13078.

11. Melaugh, W.; Gibson, B. W.; Campagnari, A. A. J. Bacteriol. 1996, 178, 564-570.

12. Schweda, E. K.; Sundstrom, A. C.; Eriksson, L. M.; Jonasson, J. A.; Lindberg, A. A. J. Biol. Chem. 1994, 269, 12040-12048.

13. Bozue, J. A.; Wang, J.; Tullius, M. V.; Gibson, B. W.; Munson, J. R. S. J. Biol. Chem. 1999, 274, 4106-4114.

14. Schweda, E. K.; Jonasson, J. A.; Jansson, P. E. J. Bacteriol. 1995, $177,5316-5321$.

15. John, C. M.; Schneider, H.; Griffiss, J. M. J. Biol. Chem. 1999, 274, 1017-1025.

16. Kniep, B.; Schakel, K.; Nimtz, M.; Schwartz-Albiez, R.; Schmitz, M.; Northoff, H.; Vilella, R.; Gramatzki, M.; Rieber, E. P. Glycobiol. 1999, 9, 399-406.
17. Prieto, P. A.; Larsen, R. D.; Cho, M.; Rivera, H. N.; Shilatifard, A.; Lowe, J. B.; Cummings, R. D.; Smith, D. F. J. Biol. Chem. 1997, 272, 2089-2097.

18. Heffernan, M.; Lotan, R.; Amos, B.; Palcic, M.; Takano, R.; Dennis, J. W. J. Biol. Chem. 1993, 268, 1242-1251.

19. Sun, S. H.; Schilling, B.; Tarantino, L.; Tullius, M. V.; Gibson, B. W.; Munson, R. S. J. Bacteriol. 2000, 182, 2292-2298.

20. Filiatrault, M.; Gibson, B. W.; Schilling, B.; Sun, S.; Munson, R. S., Jr.; Campagnari, A. A. Infect. Immun. 2000, 68, 3352-3361.

21. Campagnari, A. A.; Spinola, S. M.; Lesse, A. J.; Kwaik, Y. A.; Mandrell, R. E.; Apicella, M. A. Microb. Pathog. 1990, 8, 353-362.

22. Campagnari, A. A.; Wild, L. M.; Griffiths, G. E.; Karalus, R. J.; Wirth, M. A.; Spinola, S. M. Infect Immun. 1991, 59, 2601-2608.

23. Tsai, C. M.; Frasch, C. E. Anal. Biochem. 1982, 119, 115-119.

24. Knecht, D. A.; Dimond, R. L. Anal. Biochem. 1984, 136, 180-184.

25. Apicella, M. A.; Griffiss, J. M.; Schneider, H. Methods Enzymol. 1994, 235, 242-252.

26. Johnson, K. G.; Perry, M. B. Can. J. Microbiol. 1976, 22, 29-35.

27. Westphal, O.; Jahn, K.In Methods in Carbohydrate Chemistry; Whistler, R. L., Ed.; Academic Press Inc.: New York, 1965; 5, 83-91.

28. Helander, I. M.; Nummila, K.; Kilpelainen, I.; Vaara, M. Prog. Clin. Biol. Res. 1995, 392, 15-23.

29. Spengler, B.; Kirsch, D.; Kaufmann, R. Rapid Commun. Mass Spectrom. 1992, 6, 105-108.

30. Vestal, M. L.; Juhasz, P.; Martin, S. A. Rapid Commun. Mass Spectrom. 1995, 9, 1044-1050.

31. Mohr, M. D.; Bornsen, K. O.; Widmer, H. M. Rapid Commun. Mass Spectrom. 1995, 9, 809-814.

32. Phillips, N. J.; Apicella, M. A.; Griffiss, J. M.; Gibson, B. W. Biochemistry 1992, 31, 4515-4526.

33. Harvey, D. J. Mass Spectrom. Rev. 1999, 18, 349-450.

34. Olsthoorn, M. M. A.; Haverkamp, J.; Thomas-Oates, J. E. J. Mass Spectrom. 1999, 34, 622-636.

35. Harvey, D. J. J. Mass Spectrom. 2000, 35, 1178-1190.

36. Kuster, B.; Naven, T. J.; Harvey, D. J. J. Mass Spectrom. 1996, 31, 1131-1140.

37. Venkataraman, G.; Shriver, Z.; Raman, R.; Sasisekharan, R. Science 1999, 286, 537-542.

38. Schneider, H.; Griffiss, J. M.; Boslego, J. M.; Hitchcock, P. J.; Zahos, K. M.; Apicella, M. A. J. Exp. Med. 1991, 174, 1601-1605.

39. Spinola, S. M.; Wild, L. M.; Apicella, M. A.; Gaspari, A. A.; Campagnari, A. A. J. Infect. Dis. 1994, 169, 1146-1150.

40. Domon, B.; Costello, C. E. Glycoconj. J. 1988, 5, 397-409. 